\title{
NOMBRE DE CAS DE LĖPRE AU BRÉSIL DE 2010 À 2015
}

\section{ARTICLE ORIGINAL}

MONTEIRO, Lis Mayra Dantas¹, FACCO, Lucas², FECURY, Amanda Alves³ ARAÚJO, Maria Helena Mendonça de ${ }^{4}$, OLIVEIRA, Euzébio de ${ }^{5}$, DENDASCK, Carla Viana $^{6}$, SOUZA, Keulle Oliveira da7 ${ }^{7}$ DIAS, Claudio Alberto Gellis de Mattos ${ }^{8}$

MONTEIRO, Lis Mayra Dantas. Et al. Nombre de cas de lèpre au Brésil de 2010 à 2015. Revista Científica Multidisciplinar Núcleo do Conhecimento. An 05, Ed. 11, vol. 25, p. 62-70. novembre 2020. ISSN: 2448-0959, Lien d'accès: https://www.nucleodoconhecimento.com.br/sante/lepre-au-bresil, $\quad$ DOI: 10.32749/nucleodoconhecimento.com.br/sante/lepre-au-bresil

\section{RÉSUMÉ}

La lèpre est considérée comme une maladie infectieuse causée par la bactérie Mycobacterium leprae également connue sous le nom de Bacillus de Hansen. En plus d'être une maladie avec une évolution progressive, la lèpre dépend de la relation entre le parasite et l'hôte. L'objectif de cette étude était de montrer le nombre de cas de lèpre au Brésil entre 2010 et 2015 au Brésil. La recherche a été menée à DATASUS (http://datasus.saude.gov.br/). Avec l'avancée des politiques publiques, le nombre de cas de maladie au Brésil a diminué entre 2010 et 2015. II a une prévalence plus élevée chez les hommes et le taux le plus élevé de patients est dans

\footnotetext{
${ }^{1}$ Technique minière, à la suite de l'Institut fédéral d'Amapá (IFAP).

2 Étudiant du cours de médecine de l'Université fédérale d'Amapá (UNIFAP).

${ }^{3}$ Biomédical, Docteur en maladies tropicales, Professeur et chercheur du Cours médical de l'Université fédérale d'Amapá (UNIFAP).

${ }^{4}$ Médecin, professeur et chercheur du cours de médecine de l'Université fédérale d'Amapá (UNIFAP).

${ }^{5}$ Biologiste, PhD en maladies topiques, professeur et chercheur du cours d'éducation physique de l'Université fédérale du Pará (UFPA).

${ }^{6}$ Teologo, PhD in Psicoanalisi, ricercatore presso il Centro Ricerche e Studi Avanzati - CEPA.

${ }^{7}$ Sociologue, étudiant à la maîtrise en études anthropiques en Amazonie, membre du groupe de recherche « Laboratoire d'éducation, d'environnement et de santé » (LEMAS/UFPA).

${ }^{8}$ Biologo, Dottore in Teoria e Ricerca sul Comportamento, Professore e ricercatore presso il Corso di Laurea in Educazione Professionale e Tecnologica (PROFEPT), Istituto Federale di Amapá (IFAP).
}

$\mathrm{RC}: 68073$

Disponible en: https://www.nucleodoconhecimento.com.br/sante/lepre-au-bresil 
les grands groupes d'âge, c'est-à-dire chez les adultes. La période d'incubation de la maladie (qui peut varier de 2 à 7 ans). Dans ce contexte, plusieurs problèmes peuvent être déclenchés au niveau individuel, tels que les participations physiques débilitantes et psychologiques. Afin de réduire le nombre de cas de la maladie dans le pays et d'être en mesure de faire le diagnostic le plus tôt possible, les résultats cliniques et les tests disponibles pour cette détection devraient être analysés.

Mots-clés: Épidémiologie, lèpre, Mycobacterium leprae.

\section{INTRODUCTION}

La lèpre est considérée comme une maladie infectieuse, car elle a besoin d'un contact direct avec le bacille pour être transmise entre les personnes. Elle est causée par la bactérie Mycobacterium leprae également connue sous le nom de Bacillus de Hansen. Cette maladie n'est détectée, en moyenne, qu'entre 2 et 7 ans d'incubation et les symptômes apparaissent généralement de 11 à 16 jours. Dans cette phase symptomatique, la transmission interpersonnelle est possible, et habituellement seulement une petite partie tombe malade (BRASIL, 2002 ; OLIVEIRA et al., 2016).

La lèpre peut être classée sous deux formes : la lèpre paucibacillaire ou la lèpre multibacillllaire. Dans la variation paucibacilar, le patient a la résistance aux bacilles, présentant jusqu'à cinq lésions de peau. Bacilli sont en infériorité numérique dans le corps et ne sont pas suffisants pour la transmission à d'autres personnes. Dans ce cas, il ya la possibilité de guérir spontanément. La lèpre multibacllaire fait partie du soi-disant groupe de transmission, avec six lésions cutanées ou plus. Les malades ne présentent pas de défense contre la bactérie, les éliminant par les voies respiratoires (BRASIL, 2002; BRASIL, 2016). La contagion se produit habituellement par contact direct entre une personne sensible et un patient non traité, habituellement un membre de la famille (BRASIL, 2015).

$\mathrm{RC}: 68073$

Disponible en: https://www.nucleodoconhecimento.com.br/sante/lepre-au-bresil 
En plus d'être une maladie avec une évolution progressive, la lèpre dépend de la relation entre le parasite et l'hôte. Lorsque l'hôte dispose d'un système de défense efficace, il a tendance à ne pas manifester la maladie. Pour diverses raisons telles que la vulnérabilité socioéconomique et la vie avec de nombreuses personnes dans le même environnement, il peut atteindre des individus de différents âges et des deux sexes (BRASIL, 2002).

Des symptômes peuvent apparaître sur la peau ou le système nerveux. Sur la peau, ils sont généralement caractérisés par des lésions avec perte ou sensibilité accrue. Des taches pigmentaires, des plaques, des infiltrations, des tubercules et/ou des nodules peuvent apparaître. Dans le système nerveux, les symptômes peuvent apparaître comme des incapacités physiques et, selon le degré de la maladie, des déformations dans les extrémités du corps (BRASIL, 2002; FREITAS, 2015).

Le traitement de la lèpre se fait avec la combinaison de plusieurs médicaments, appelés polychimiothérapie ou MDT. Pour les patients atteints de la forme Paucibacillar, deux types d'antibiotiques sont utilisés. Pour ceux qui ont une forme multibacillure, trois types d'antibiotiques sont recommandés (BRASIL, 2002; FREITAS, 2015).

Selon le ministère de la Santé, le nombre de nouveaux cas au Brésil au cours des années étudiées était de 193021 patients. (BRASIL, 2017).

\section{OBIETTIVO}

Afficher le nombre de cas de lèpre au Brésil entre 2010 et 2015 au Brésil.

\section{MÉTHODE}

La recherche a été effectuée à DATASUS (http://datasus.saude.gov.br/), et les données sur la lèpre ont été recueillies à partir des étapes suivantes : dans l'onglet « Accès à l'information », "Information sur la santé (TABNET) » a été sélectionnée et

RC: 68073

Disponible en: https://www.nucleodoconhecimento.com.br/sante/lepre-au-bresil 
immédiatement après avoir cliqué sur "Épidémiologique et morbidité ». Sur la page suivante en cliquant sur "Cas de lèpre - Depuis 2001 (SINAN) », l'option « Lèpre depuis 2001 " a été choisie, et dans le cadre géographique, nous avons opté pour "Brésil par région et unité de la Fédération". Les données ont été recueillies de 2010 à 2015, en choisissant : en ligne les options "Année diagnostique », "Région ", "Sexe », " Groupe d'âge », " Mode de détection », " Évaluation diagnostique » et "Lésion cutanée ". Dans le champ de colonne, l'option " Non actif " et dans le contenu de l'expression « Patients for Care » ont été utilisés pour les options de ligne ci-dessus. Après la sélection, ces données ont été compilées dans l'outil Excel, la composante Office de Microsoft Corporation. Recherche bibliographique réalisée dans le laboratoire de l'Institut fédéral d'éducation, de science et de technologie d'Amapá, campus de Macapá, situé à: Rodovia BR 210 KM 3, s/n - Bairro Brasil Novo. CEP: 68.909-398, Macapá, Amapá, Brasil.

\section{RÉSULTATS}

La figure 1 montre le nombre de cas de lèpre au Brésil entre 2010 et 2015. On observe qu'au cours des dernières années, il y a eu une diminution du nombre de cas, avec une légère augmentation du nombre de cas en 2014 par rapport à 2013.

RC: 68073

Disponible en: https://www.nucleodoconhecimento.com.br/sante/lepre-au-bresil 
Figure 1 Nombre de cas de lèpre au Brésil entre 2010 et 2015.

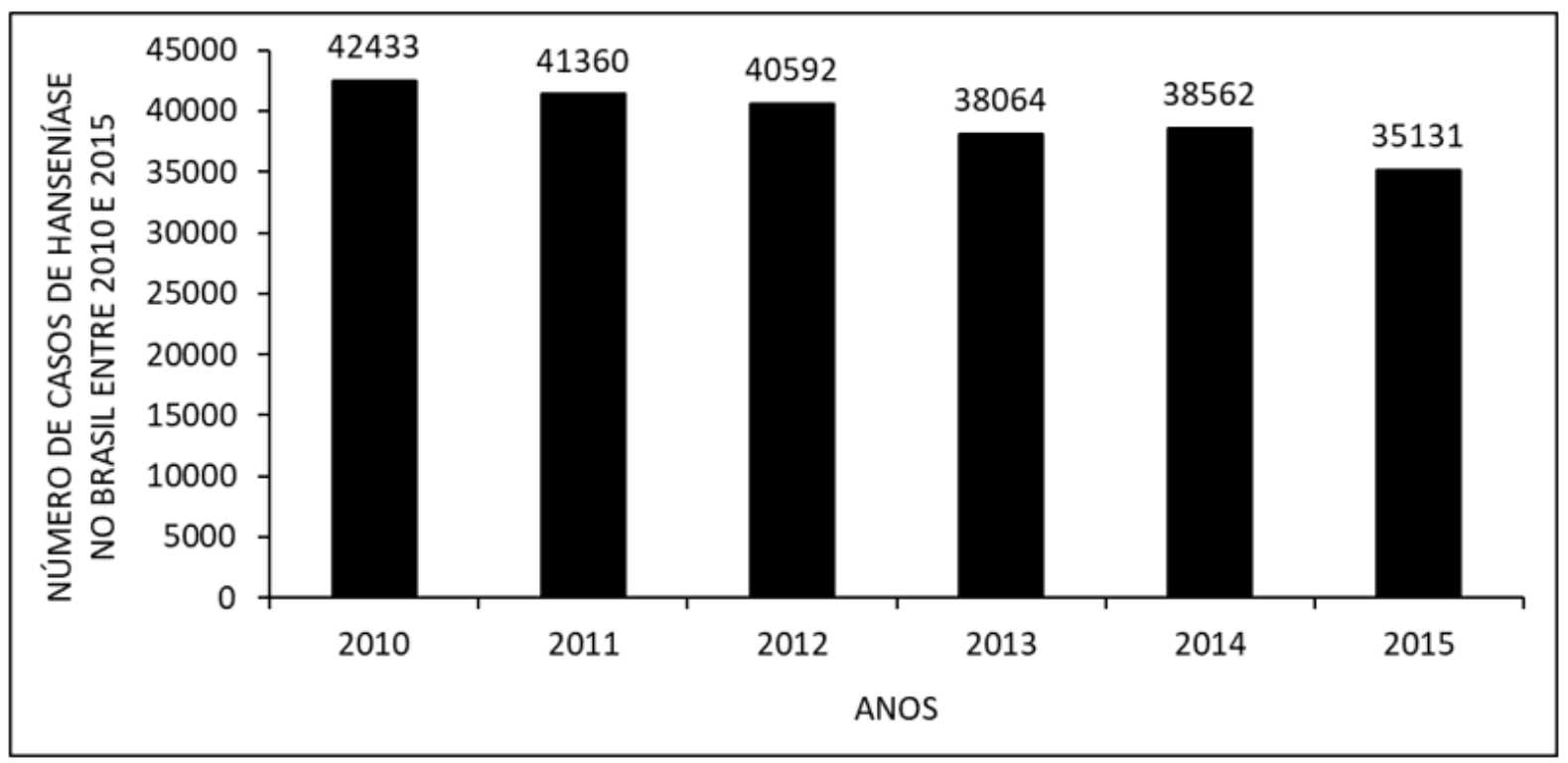

La figure 2 montre le nombre de cas de lèpre au Brésil entre 2010 et 2015, par régions du pays. Les données montrent que le plus grand nombre de visites a lieu dans le Nord-Est et la minorité dans le Sud.

RC: 68073

Disponible en: https://www.nucleodoconhecimento.com.br/sante/lepre-au-bresil 
La figure 2 montre le nombre de cas de lèpre au Brésil entre 2010 et 2015, par régions du pays.

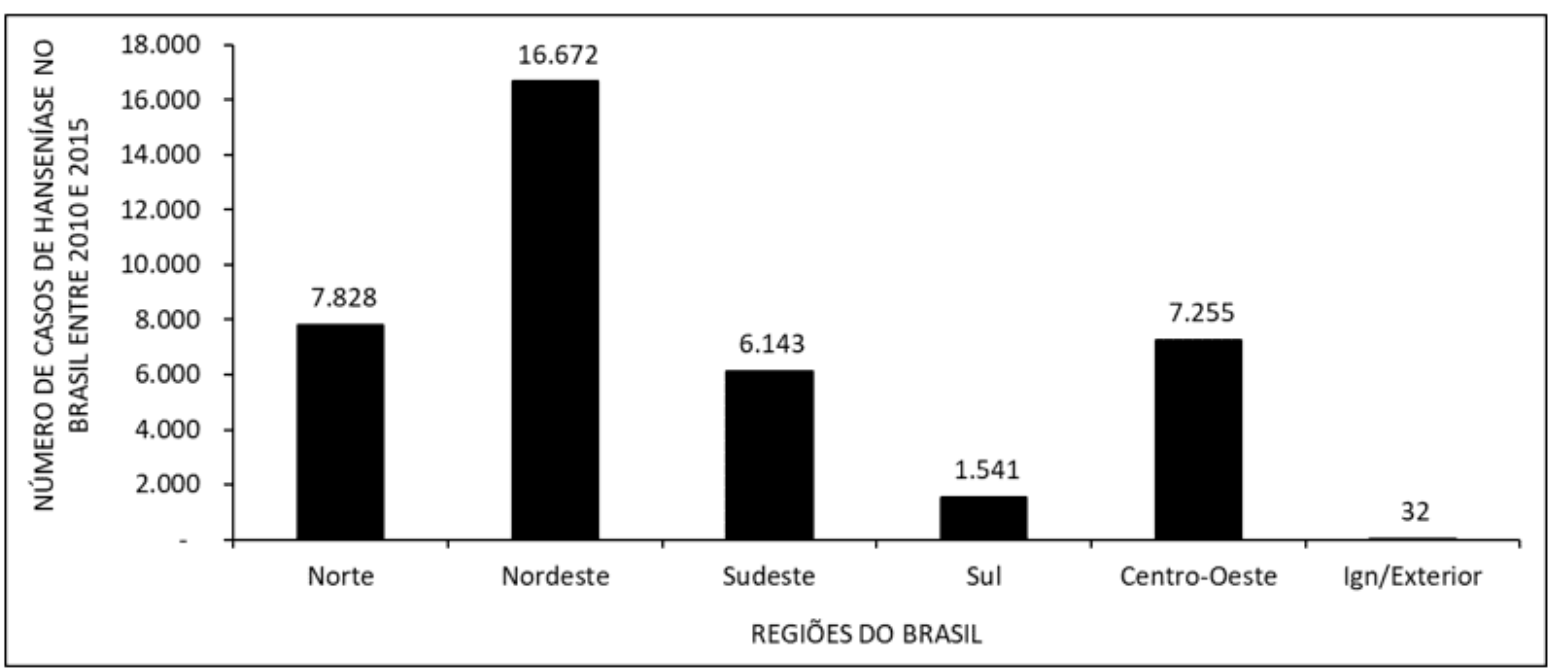

La figure 3 montre le nombre de cas de lèpre au Brésil entre 2010 et 2015, selon le sexe. Lorsqu'on observe qu'il y a un plus grand nombre d'occurrences chez les hommes par rapport aux femelles.

RC: 68073

Disponible en: https://www.nucleodoconhecimento.com.br/sante/lepre-au-bresil 
La figure 3 montre le nombre de cas de lèpre au Brésil entre 2010 et 2015, selon le sexe.

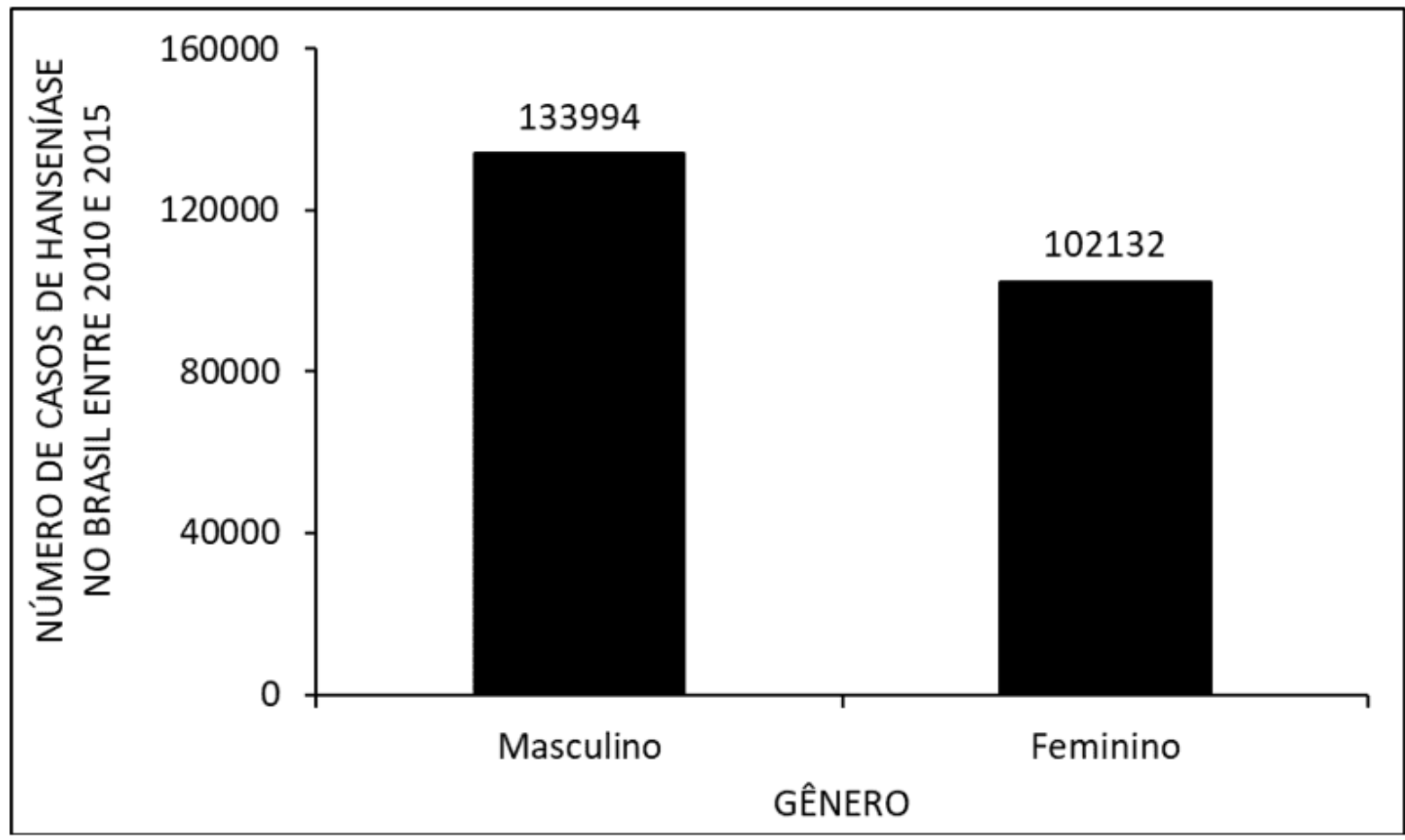

La figure 4 montre le nombre de cas de lèpre au Brésil entre 2010 et 2015, selon le groupe d'âge. Lorsqu'un taux plus élevé d'occurrence de la maladie est noté chez les adultes âgés de 20 à 64 ans, et plus faible chez les enfants âgés de 1 à 9 ans et les personnes âgées de 80 ans ou plus.

RC: 68073

Disponible en: https://www.nucleodoconhecimento.com.br/sante/lepre-au-bresil 
La figure 4 montre le nombre de cas de lèpre au Brésil entre 2010 et 2015, selon le groupe d'âge.

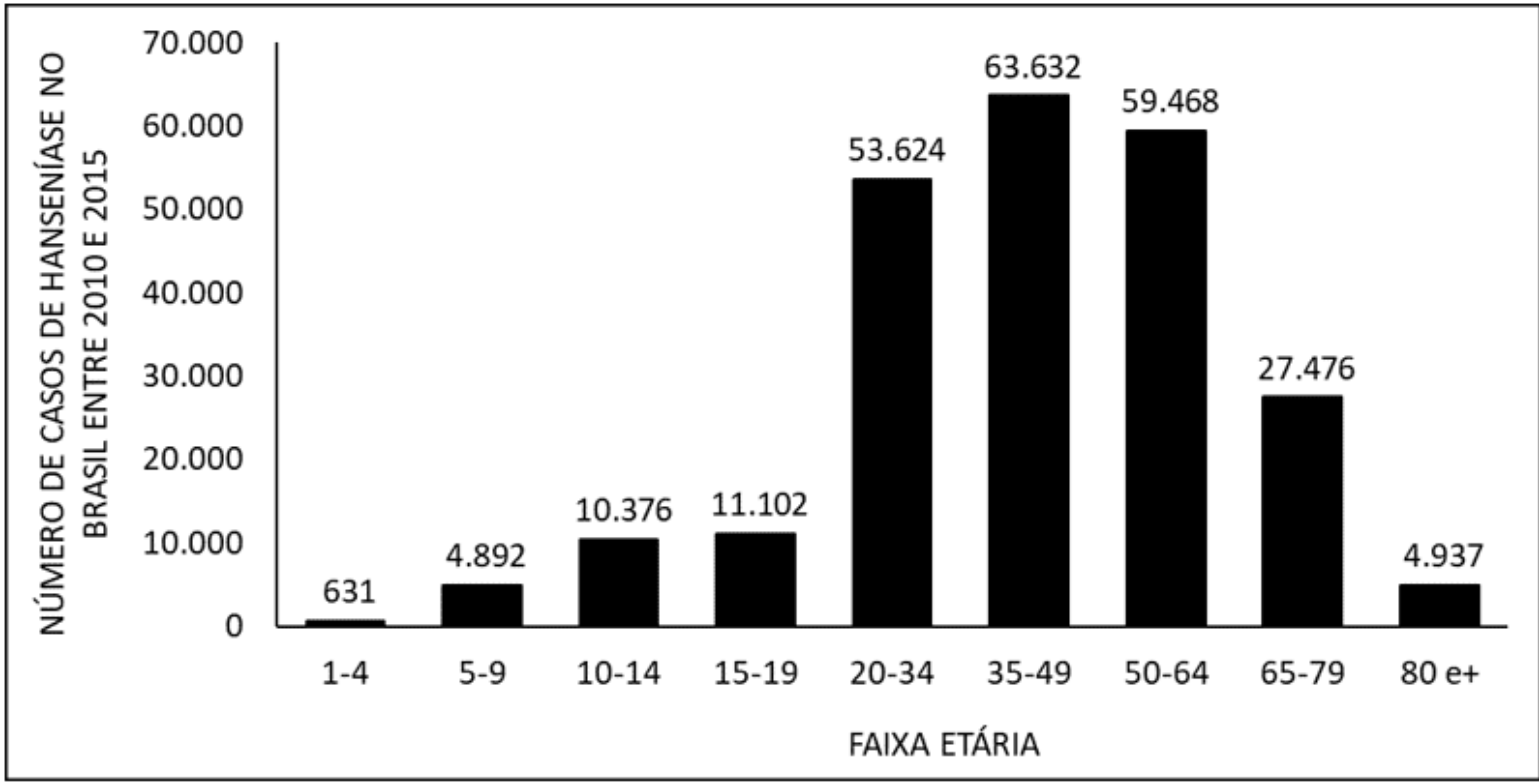

La figure 5 montre le nombre de cas de lèpre au Brésil entre 2010 et 2015, selon le mode de détection. Le principal mode de détection de la maladie est le renvoi, suivi d'une demande spontanée.

RC: 68073

Disponible en: https://www.nucleodoconhecimento.com.br/sante/lepre-au-bresil 
La figure 5 montre le nombre de cas de lèpre au Brésil entre 2010 et 2015, selon le mode de détection.

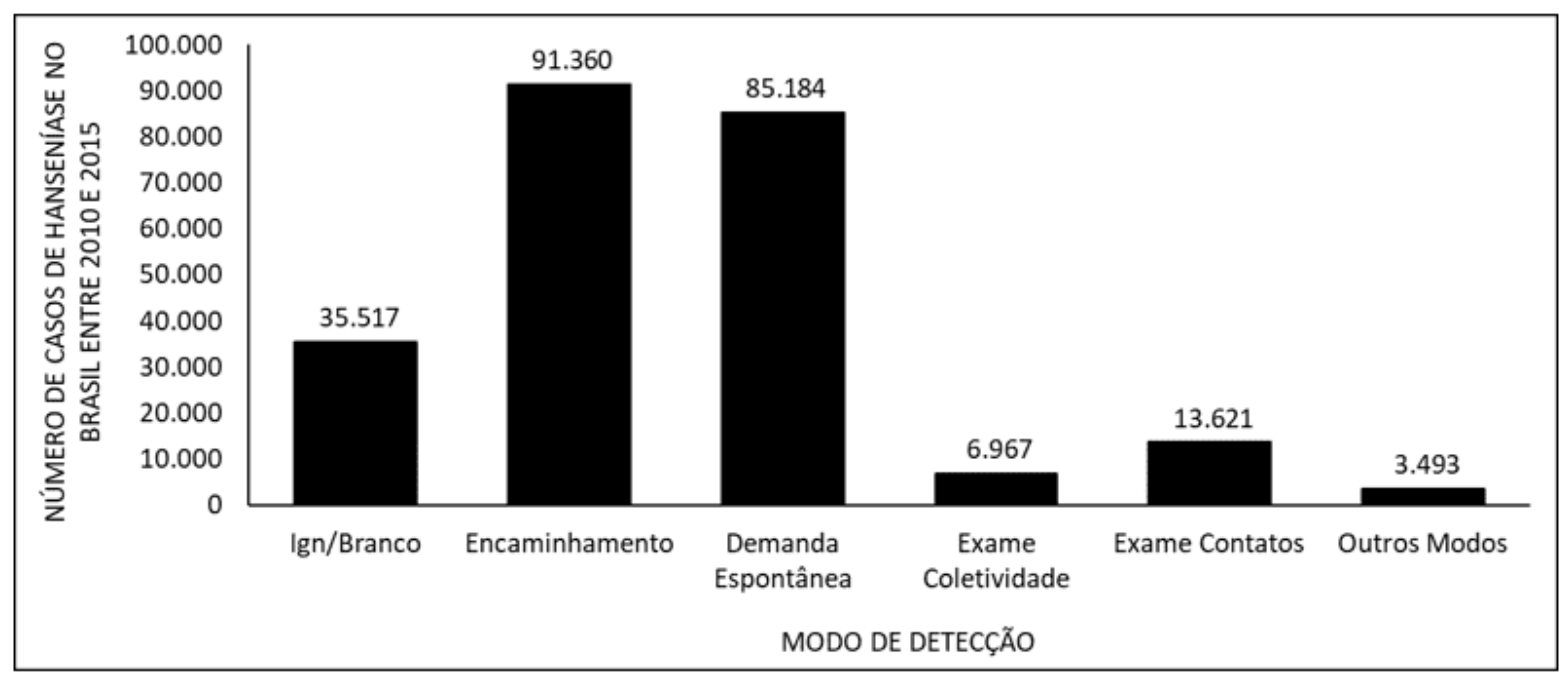

La figure 6 montre le nombre de cas de lèpre au Brésil entre 2010 et 2015, en raison de types de lésions cutanées. Les données montrent que la plupart des patients ont 2 à 5 lésions cutanées et la minorité ne présente aucune lésion.

RC: 68073

Disponible en: https://www.nucleodoconhecimento.com.br/sante/lepre-au-bresil 
La figure 6 montre le nombre de cas de lèpre au Brésil entre 2010 et 2015, par type de lésion cutanée.

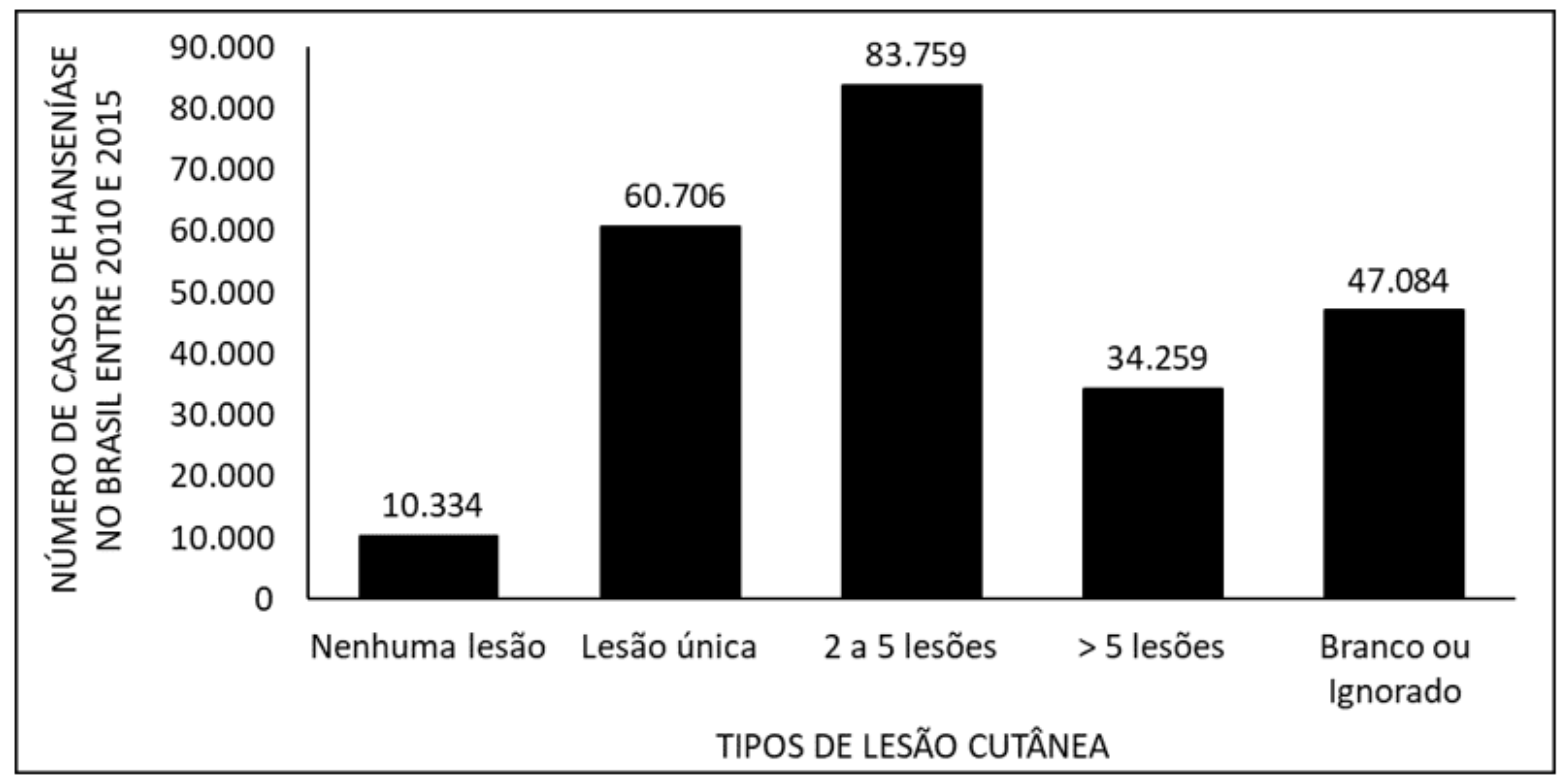

La figure 7 montre le nombre de cas de lèpre au Brésil entre 2010 et 2015, par évaluation diagnostique. Les données montrent que la plupart des cas appartiennent à la catégorie zéro et la minorité à la catégorie II de la maladie.

RC: 68073

Disponible en: https://www.nucleodoconhecimento.com.br/sante/lepre-au-bresil 
La figure 7 montre le nombre de cas de lèpre au Brésil entre 2010 et 2015, par évaluation diagnostique.

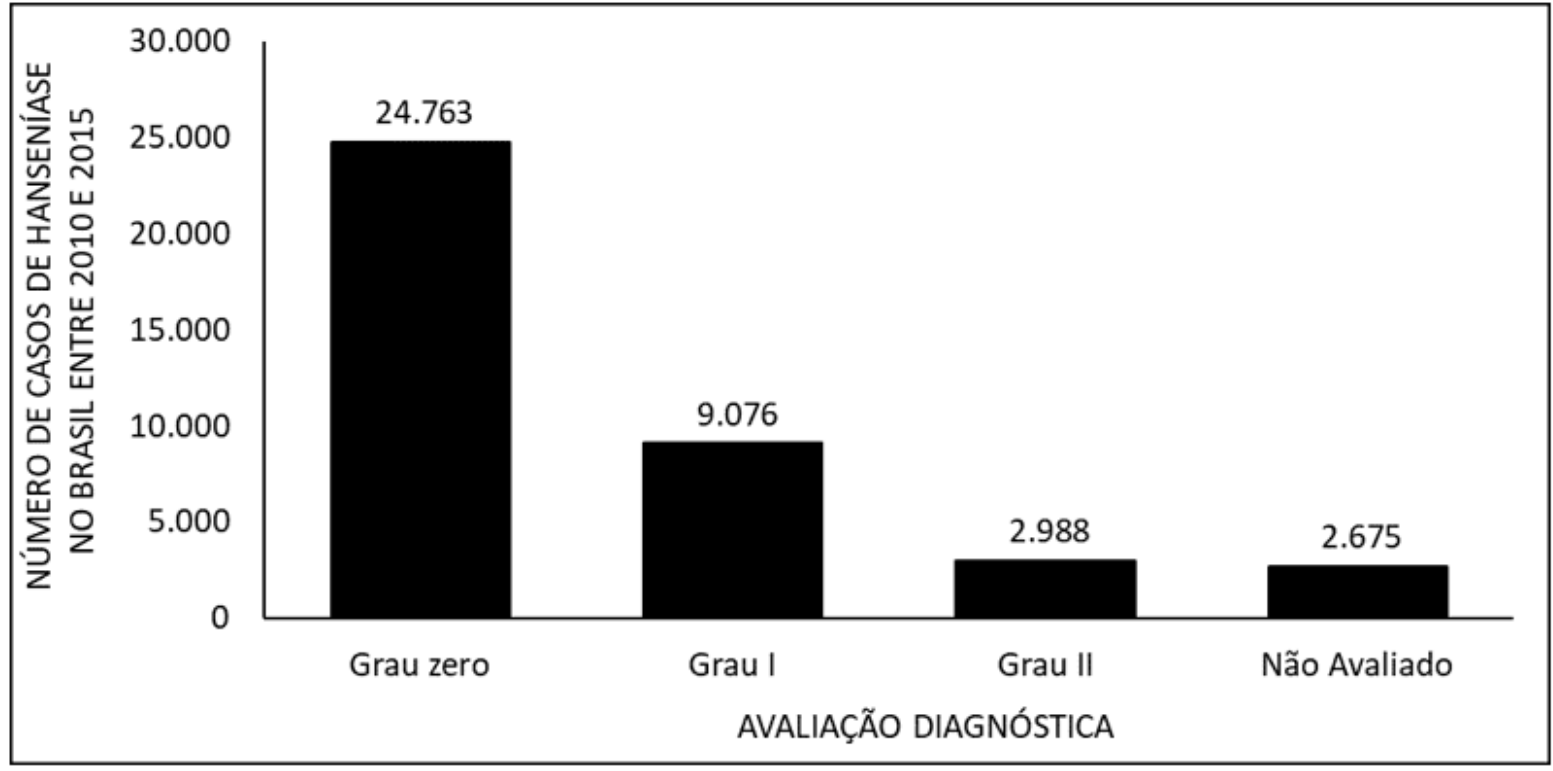

\section{DISCUSSION}

Entre 2010 et 2015, le nombre de patients atteints de lèpre a diminué, avec une légère augmentation en 2014 (figure 1). Les données sont corroborées par la littérature. La diminution s'est probablement produite par la mise en œuvre de politiques publiques pour faire face à la maladie, comme le diagnostic précoce et le traitement réussi, qui mène à la guérison. L'augmentation du nombre de cas en 2014 est probablement due à la campagne de détection de nouveaux cas de la maladie (BRASIL, 2015; 2017; 2017a).

Le nombre de cas de lèpre montre plus de visites dans le nord-est et moins dans la région sud (figure 2). Selon la littérature, ce nombre élevé de personnes infectées dans le Nord-Est est probablement dû au taux élevé de transmission, qui se produit dans les saisons festives. À une époque où les unités de santé de base n'agissent pas. Dans la région sud, la cause probable du faible indice des patients se réfère à la distance entre les services de santé et le patient, entravant l'accès et contribuant à l'évolution de la maladie, à un degré élevé d'incapacité physique (ROCHA, 2015).

$\mathrm{RC}: 68073$

Disponible en: https://www.nucleodoconhecimento.com.br/sante/lepre-au-bresil 
Selon le sexe, entre 2010 et 2015 , l'incidence de la maladie chez les hommes était plus élevée que celle des femmes (figure 3). Lorsque l'on compare les données avec la littérature, on observe que la cause probable de ce taux élevé est due au fait que les hommes sont plus exposés à la maladie que les femmes, en prenant le temps de chercher des services de santé et d'abandonner le traitement avant de guérir. Cela le rend susceptible de développer la déformation caractéristique de la maladie (ARAGOSO et al., 2014; ARANTES et al., 2010).

Au niveau de l'occurrence, les individus du groupe d'âge plus élevé prédominent parmi les infectés. L'un des facteurs les plus répandus pour cet aspect est le temps d'incubation de la bactérie (Mycobacterium leprae), qui peut s'étendre de deux à sept ans et, par conséquent, se présenter à l'âge adulte et, avec cela, finit par aboutir à d'éventuelles implications psychologiques, puisque, avec l'apparition de problèmes physiques - amplifiés par le retard du diagnostic - le patient peut se trouver incapable d'accomplir son activité professionnelle et, par conséquent, la difficulté de contribuer économiquement dans l'environnement familial. De plus, en cas de cas familiaux, les risques de maladie chez les enfants peuvent être accrus (VELÔSO, 2018).

Pour le diagnostic de lèpre, et sa classification et mise en scène conséquentes, plusieurs aspects sont analysés. Ces facteurs peuvent varier selon la classification utilisée, dont deux sont les principaux, ridley et Jopling, basés sur des caractéristiques histopathologiques, cliniques, bacilloscopiques et immunologiques; la Classification de l'Organisation mondiale de la Santé (OMS) utilise l'indice bacilloscopique dans sa classification, établissant que, s'il y a un maximum de cinq lésions au niveau cutané et au tronc nerveux, la lèpre paucibacilaire l'est. Dans le cas où il y a plus de cinq lésions, au niveau cutané et au tronc nerveux, il y a alors la lèpre multibacllaire. Toutefois, si la microscopie par frottis d'expectoration est disponible chez le patient et que le résultat positif est atteint, la classification est automatiquement basée sur la multibacillure (LASTÓRIA et ABREU, 2012).

$\mathrm{RC}: 68073$

Disponible en: https://www.nucleodoconhecimento.com.br/sante/lepre-au-bresil 
Selon la forme de manifestation de lèpre, plusieurs participations peuvent être physiquement présentes chez l'individu infecté dont le bacille est actif, parmi eux : lésions granulomatous (qui peuvent survenir sous différentes formes, telles que nodules ou papules), œdème, symptômes provoqués lorsque les troncs nerveux sont affectés (comme l'atrophie musculaire, faiblesse et douleur), problèmes immunitaires, faiblesses sensorielles, motrices, processus inflammatoires aigus et systémiques et autres implications pour le corps (PESSOAS , 2019).

La lèpre, afin de ne pas déclencher une implication grave pour l'individu, doit être diagnostiquée tôt, en utilisant la logique clinique et les tests recommandés. Si le diagnostic se produit tard, les implications peuvent être plus grandes. En outre, l'épidémiologie et son contrôle sont nécessaires pour une large connaissance de la situation de prévalence de la maladie, reflétant ainsi le nombre de cas, parce que, si le contrôle n'est pas efficace, il peut y avoir une plus grande diffusion de la maladie et, par conséquent, une augmentation significative du nombre de cas. La recherche d'aide médicale au stade initial de la maladie aboutit à la prévalence des cas appartenant à la catégorie zéro (dans lesquels les segments musculaires et leurs sensibilités respectives sont préservés, caractérisant, en grande partie, un bon pronostic du patient) (PESSOA, 2019; BRASIL, 2018).

\section{CONCLUSION}

Avec l'avancée des politiques publiques de lutte contre la lèpre, basées sur le diagnostic et le traitement précoces, le nombre de cas de la maladie au Brésil, au niveau majoritaire, a diminué entre 2010 et 2015.

La lèpre a une prévalence plus élevée chez les hommes, et la cause probable de cette réalité est la plus grande exposition des hommes à cette pathologie, puisque la recherche de services de santé est moins complète et, en outre, l'abandon plus important du traitement, entraînant l'avancée de la maladie et l'émergence conséquente de faiblesses organiques de l'individu.

RC: 68073

Disponible en: https://www.nucleodoconhecimento.com.br/sante/lepre-au-bresil 
En outre, le taux le plus élevé de patients est dans les groupes d'âge plus grands, c'est-à-dire chez les adultes, et le principal facteur en est la période d'incubation de la maladie (qui peut varier de 2 à 7 ans). Dans ce contexte, plusieurs problèmes peuvent être déclenchés au niveau individuel, tels que les participations physiques débilitantes et psychologiques, et la famille, car, avec l'émergence de faiblesse, des difficultés économiques peuvent survenir en raison de la perte d'activité professionnelle rémunérée.

Afin de réduire le nombre de cas de la maladie dans le pays et d'être en mesure de faire le diagnostic le plus tôt possible, les résultats cliniques et les tests disponibles pour cette détection devraient être analysés. Si le patient peut avoir son diagnostic fait au stade tôt de la maladie, il y a habituellement un bon pronostic, et sa santé organique peut être considérablement préservée, maintenant la sensibilité et l'intégrité de ses segments de muscle.

\section{RÉFÉRENCES}

ARAGOSO, I.; CARVALHO, R.M.B.; SOUSA, C.M.; VIEIRA, G.D. Hanseníase em Rondônia: incidência e características dos casos notificados, 2001 a 2012. Epidemiologia e Serviços de Saúde, v.23, n.2, Brasília, jun. 2014.

ARANTES, C.K.; FILIPE, M.S.; GARCIA, M.L.R.; NARDI, M.T.; PASCHOAL, V.D.A. Avaliação dos serviços de saúde em relação ao diagnóstico precoce da hanseníase. Epidemiologia e Serviços de Saúde, v.19, n.2, Brasília, jun. 2010.

BRASIL. Ministério da Saúde. Secretaria de Políticas de Saúde. Departamento de Atenção Básica. Guia para o controle da hanseníase. 1aㅡ ed., Brasília (DF): Ministério da Saúde, $2002 . \quad$ Disponível em: <http://bvsms.saude.gov.br/bvs/publicacoes/guia_de_hanseniase.pdf> Acesso em: 24 ago 2017.

$\mathrm{RC}: 68073$

Disponible en: https://www.nucleodoconhecimento.com.br/sante/lepre-au-bresil 
BRASIL. Ministério da Saúde. Secretaria de Vigilância em Saúde. Departamento de Vigilância das Doenças Transmissíveis. Diretrizes para vigilância, atenção e eliminação da Hanseníase como problema de saúde pública: manual técnicooperacional. 1aㅡ. ed., Brasília (DF): Ministério da Saúde, 2016. Disponível em: <http://www.saude.pr.gov.br/arquivos/File/DiretrizesdoManuaTcnicoOperacionaldeH ansenase.pdf> Acesso em: 05 set 2017.

BRASIL. Ministério da Saúde. Secretaria de Vigilância em Saúde. Departamento de Vigilância das Doenças Transmissíveis. Eliminar a hanseníase é possível: um guia para os municípios. Brasília (DF): Ministério da Saúde, 2015. Disponível em: <http://bvsms.saude.gov.br/bvs/publicacoes/eliminar_hanseniase_possivel_versao_p reliminar.pdf> Acesso em: 05 set 2017.

BRASIL. Boletim Epidemiológico. Brasilía DF. Ministério da Saúde. 49, n. 4, 12p. 2018.

FREITAS, A.A. Resposta imune celular e humoral a proteínas recombinantes do Mycobacterium leprae em pacientes com outras dermatoses. Tese (Doutorado) Universidade Federal de Goiás, Instituto de Patologia Tropical e Saúde Pública, Program de Pós Graduação em Medicina Tropical e Saúde Pública, Goiânia, 2015. Disponível em: $<\mathrm{https}: / /$ posstrictosensu.iptsp.ufg.br/up/59/o/AlinedeAraujoFreitas_2015_vers\%C3\%A3ofinal. pdf $>$ Acesso em: 12 set 2017.

LASTÓRIA, J. C; ABREU, M. A. M. M. Hanseníase: diagnóstico e tratamento. Diagn Tratamento, v. 17, n. 4, p. 173-179, 2012.

OLIVEIRA, Ciane Martins de; et. al. A evolução da assistência ao paciente com Hanseníase: dos leprosários à poliquimioterapia. Revista Científica Multidisciplinar Núcleo do Conhecimento. Ano 01, Vol. 06, Ed. 08, pp. 68-80. Agosto de 2016. ISSN: 2448-0959

$\mathrm{RC}: 68073$

Disponible en: https://www.nucleodoconhecimento.com.br/sante/lepre-au-bresil 
PESSOA, M. M. S. F. S. Hanseníase no Brasil: Uma revisão literária, nos anos de 2014 a 2019. 45p. Trabalho de Conclusão de Curso (Graduação de Farmácia) Universidade Federal do Rio Grande do Norte, Rio Grande do Norte, Natal, 2019.

ROCHA, A.C.A.A. Análise da sazonalidade da incidência de hanseníase segundo regiões geográficas, climas e biomas do Brasil. 2015. 82 f. Dissertação (Mestrado em Ciências Ambientais) - Universidade do Estado de Mato Grosso, Programa de Pós-Graduação em Ciências Ambientais, Cáceres/MT.

VELÔSO, D. S. Perfil clínico-epidemiológico da hanseníase no estado do Piauí, no período de 2009 a 2016. 127p. Dissertação (Mestrado em Medicina Tropical) Instituto Oswaldo Cruz, Piauí, Teresina, 2018.

Soumis : Novembre 2020.

Approuvé : Novembre 2020.

RC: 68073

Disponible en: https://www.nucleodoconhecimento.com.br/sante/lepre-au-bresil 